\title{
A new method of predicting the saturation pressure of oil reservoir and its application
}

\author{
Guoyi Yu ${ }^{a}$, Feng Xu ${ }^{a, b, *}$, Yingzhi Cui ${ }^{c}$, Xiangling $\mathrm{Li}^{b}$, Chujuan Kang ${ }^{b}$, \\ Cheng Lu ${ }^{a}$, Siyu $\mathrm{Li}^{d}$, Lin Bai ${ }^{a}$, Shuheng Du ${ }^{e}$ \\ a China National Oil and Gas Exploration and Development Company Ltd. (CNODC), Beijing, 100034, China \\ ${ }^{\mathrm{b}}$ Research Institute of Petroleum Exploration \& Development, Co., Ltd. (RIPED), CNPC, Beijing, 100083, China \\ c The University of New South Wales, School of Mineral Energy and Resource Engineering, Sydney, 2052, Australia \\ d China Petroleum Technology and Development Corporation, Beijing, 100028, China \\ e State Key Laboratory of Nonlinear Mechanics, Institute of Mechanics, Chinese Academy of Sciences, Beijing, \\ 100190, China
}

\section{H I G H L I G H T S}

- Machine learning could be used to determine oil saturation pressure.

- The new method is simple and accurate for rapid calculation.

- The new method lays a foundation for fossil hydrogen energy development.

\section{A R T I C L E I N F O}

\section{Article history:}

Received 19 June 2020

Received in revised form

27 July 2020

Accepted 5 August 2020

Available online 10 September 2020

Keywords:

Oil reservoir

Saturation pressure

Random forest

Decision tree

ANN

Empirical formula

\begin{abstract}
A B S T R A C T
Saturation pressure is a vital parameter of oil reservoir which can reflect the oilfield characteristics and determine the oilfield development process, and it is determined by experiments in the laboratory in general. However, there was only one well with saturation pressure test in this target reservoir, and it is necessary to determine whether this parameter is right or not.

In this work, we present a new method for quickly determining saturation pressure using machine learning algorithms, including random forest regressor (RF), support vector machine (SVM), decision trees (DT), and artificial neural network (ANN or NN). Using these approaches, saturation pressure was obtained by using the initial solution gas-oil ratio (GOR), temperature, API gravity and other reservoir-fluid data available in the oilfields. Compared with the empirical formula for saturation pressure calculation, the calculated result shows that the accuracy given from machine learning is higher than that from other formulas at home and abroad, and has a good match with the lab test. On the basis of the calculated saturation pressure, it can determine whether the reservoir enters into the stage of dissolved gas drive or not, which also provides the basis for maintaining the reservoir pressure by water injection in advance, rational development decision-making and work over measures.

This approach above can provide technical guidance for predicting the saturation pressure in the development of different kinds of reservoirs, including the sandstone reservoirs and carbonate reservoirs.
\end{abstract}

\footnotetext{
* Corresponding author. China National Oil and Gas Exploration and Development Company Ltd. (CNODC), Energy and Fuel Exploitation, No.6, Fuchengmen North Street, Xicheng District, Beijing, China.

E-mail address: xufeng01@cnpcint.com (F. Xu). 
(C) 2020 Hydrogen Energy Publications LLC. Published by Elsevier Ltd. All rights reserved.

\section{Introduction}

The liquid hydrocarbon inside the earth is a type of significant fossil energy, which can form various types of fuel after different processes [1-4]. Liquid hydrocarbon exists in various rocks, and its properties such as pressure, phase state and content are all affected by pores, mineral types and contents. A series of studies on the heterogeneities of oil-bearing reservoirs by $\mathrm{Du}$ and his collaborators have provided the irreplaceable theoretical and experimental basis for the occurrence and exploitation of liquid hydrocarbons which is worthy of our great attention [5-9].

Liquid hydrocarbon usually contains some natural gas in solution in their original condition, and the saturation pressure is the pressure at which this natural gas begins to come out of the solution and form bubbles. For oils, it equals to the bubble point. For condensates, it equals to the dew point. Saturation pressure is one of the important reservoir parameters, which can reflect the reservoir characteristics and determine the process of oilfield development. Generally, there are three methods to determine it: (a) sample in the downhole liquid hydrocarbon reservoir and measure it in the laboratory; (b) sample on the ground and determine it at the simulated formation conditions; (c) calculate it by fitting fluid properties parameters and relevant data [10]. The first two methods both increase the cost of oil companies and reduce liquid hydrocarbon production because sampling would shut down the producer. As a result, most wells do not have saturation pressure data, which bring inconveniences to reservoir performance analysis and development decision-making. In addition, reservoir fluids properties vary with depth along the wellbore, there are relatively large errors when using the samples from subsurface and surface sampling to restore them at the formation condition and measure the saturation pressure. It is not possible to obtain crude oil saturation pressure using (a) and (b) for wells that have been put into production and will be produced. For this reason, reservoir engineers are trying to use a large amount of laboratory data to find an indirect method that could obtain the saturation pressure of crude oil.

At present, there are many methods used in the literature, but the experience has shown that these methods are still not ideal. Traditionally, empirical correlations (Standing, Mahmound, Al-Marhoun et al., Glaso, Petrosky-Farshad, and Vasquez-Beggs) are used for saturation pressure estimation by inputting the initial solution gas-oil ratio (GOR), temperature, API gravity and other reservoir-fluid data available [11-14]. In the past few years, machine learning has achieved great success in various application fields. With the emergence of some new application modes, it continues to open up new opportunities in the oil and gas industry. Now it is used in exploration, digital core reconstruction, stratum recognition, drilling, reservoir engineering, production operations, pipeline transportation, maintenance and anything in between [15-18]. Many researchers also consider using machine learning to predict the saturation pressure, including linear and non-linear models. Artificial neural network becomes popular in predicting PVT properties, and has a long history from the late 1990s. Tree-based ensemble regressors have robustness and are capable to maintain good accuracy for small datasets. So, we choose to utilize the popular and supervised machine learning algorithms: random forest (RF), support vector machine (SVM), decision tree (DT) and artificial neural network (ANN or NN) in this study. However, these studies have some limitations, such as a limited range of pressure and temperature, restricted in some geological area. So, we collect and use a large range of parameters in our research [19-22].

What is more, our target oilfield is a thick sandstone reservoir with medium-high porosity and medium-high permeability in a west African basin. The oilfield has been produced by natural energy for about 4 years, and it began to produce gas because of low reservoir pressure in the structural high two years ago. To make reasonable reservoir development technical policies and determine when the reservoir enters the dissolved gas flooding stage, we need to know the saturation pressure as accurately as possible. However, there was only one well with saturation pressure test in this reservoir, and the thick reservoir has a great heterogeneity. The measured saturation pressure may not represent the character of the full reservoirs.

In this research, we utilized the popular and supervised machine learning algorithms (random forest, support vector machine, decision tree and artificial neural network) to get a more accurate and stable prediction of saturation pressure. A brief description of the random forest theories and other three machine learning algorithms is first presented. Then, the methodology of machine learning models for saturation pressure calculation is illustrated. Finally, the result from machine learning algorithms and the empirical formula are analyzed, and its application is also evaluated in the target reservoir for rational development decision-making.

\section{Methodology}

\section{Random forest}

Random Forest (RF) is a supervised learning algorithm and can be used for both classification and regression problems. The algorithm creates a forest and makes it random to some extent. Forest is an ensemble of decision trees, mostly trained with the "bagging" method, which is a combination of learning models which increases the overall result. The larger the number of trees, the more accurate the result [23-25]. 


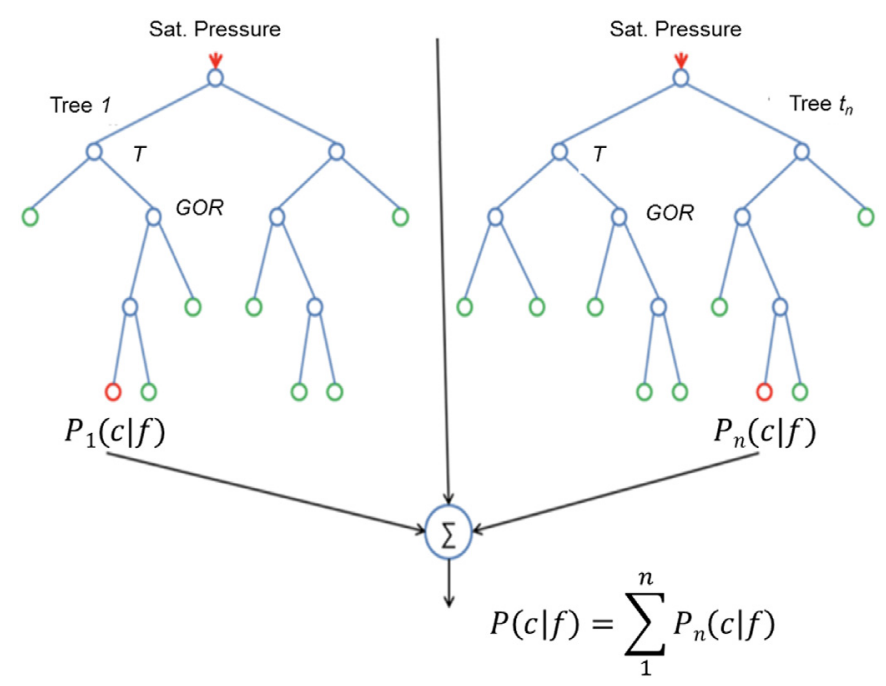

Fig. 1 - Flowchart of Decision Trees used in Random Forest.

A tree-like graph is utilized to show the possible consequences (Fig. 1). We input a training dataset with targets and features into the decision tree, it will form some set of rules. These rules can be used to perform predictions. To predict saturation pressure, we collect the initial solution gasoil ratio, temperature, API gravity and other reservoir-fluid data as the input. Then, through the decision tree algorithm, the rules can be generated. We can then input the new reservoir fluid data available and get the calculated saturation pressure. Information gain and Gini index calculations are used in the process of calculating these nodes and forming the rules. The whole process of random forest can be seen in the big data website and data science books in detail.

The following are the main steps of the whole random forest: At the beginning, load the collected data into a Pandas dataframe. After the quality check of this input, we can split the data set into training sets and test sets, about pseudorandomly $90 \%-10 \%$ split. We use the training set to train the model and do some optimization. Finally, use the test sets to evaluate model performance.

\section{Other machine learning methods}

Besides RF algorithm, we considered another three learning methods, which are Support Vector Machine (SVM) and Decision Tree (DT) and Artificial neural network (ANN). In each model, we use regularization methods to limit overfitting problems in this research.

SVM is also a type of supervised learning that is mostly used in regression and pattern recognition purposes [26]. The algorithm depends on soft margin hyper-plane and stands on kernel neuron function which definitely allows the projection to higher planes and is able to solve more complicated and nonlinear problems. The projection feature in SVM means how similar or the parameter is and it determines the degree of over-lap between the different parameters. Also, it affects

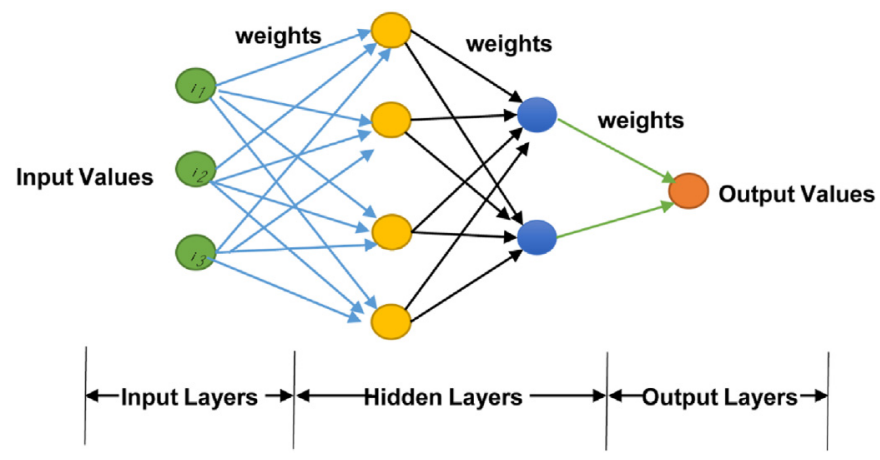

Fig. 2 - Sample artificial neural network architecture. 


\section{Table 1 - Data source used in the model.}

\begin{tabular}{lll} 
Oilfield location & Count & Source \\
\hline Global & 385 & Ref. [29] \\
Nigeria Delta & 23 & Ref. [30] \\
Global & 195 & Ref. [31] \\
UAE & 51 & Ref. [32] \\
Pakistan & 185 & Ref. [33] \\
Malaysia & 93 & Ref. [34] \\
Alaska & 8 & Ref. [35] \\
West Africa & 37 & Unpublished \\
Total & 977 & $/$
\end{tabular}

the classifications and clustering process based on the degree of overlap. SVM applications can also be found in many fields like petroleum industry, medical, business, civil, and electrical engineering.

Decision tree is one of the frequently and widely used supervised machine learning methods that can perform both classification and regression tasks [27]. For each attribute in the dataset, the decision tree algorithm forms a node, where the most important attribute is placed at the root node. To evaluate results, we start at the root node and work way down the tree by following the corresponding node that meets our condition. This process continues until a leaf node is reached, which contains the prediction or the outcome of the decision tree. The optimization process of training of decision trees is based on the purity or accuracy of prediction on training data.

Artificial neural network (ANN) is a kind of machine learning algorithms that mimic human being's biological neural system for regression or classification problem. The
Table 2 - Data range of the fluid properties.

\begin{tabular}{llllll} 
Fluid properties & Mean & STD & Min & Max & Median \\
\hline $\mathrm{T}\left({ }^{\circ} \mathrm{F}\right)$ & 192.65 & 50.93 & 69.98 & 341.60 & 187.00 \\
$\mathrm{Rs}(\mathrm{SCF} / \mathrm{STB})$ & 513.07 & 448.46 & 8.61 & 3298.70 & 384.00 \\
$\mathrm{GG}$ & 1.01 & 0.42 & 0.17 & 3.44 & 0.85 \\
$\mathrm{API}\left({ }^{\circ}\right)$ & 34.74 & 10.26 & 1.90 & 56.80 & 37.4 \\
$\mathrm{~Pb}(\mathrm{psi})$ & 1788.6 & 1244.5 & 79.0 & 8333.6 & 1496.9 \\
\hline
\end{tabular}

Table 3 - Input data for validation.

\begin{tabular}{ll} 
PVT parameter & Value \\
\hline $\mathrm{T}\left({ }^{\circ} \mathrm{C}\right)$ & 62.1 \\
$\mathrm{P}(\mathrm{psi})$ & 1420 \\
$\mathrm{R}_{\mathrm{s}}(\mathrm{SCF} / \mathrm{STB})$ & 17.0 \\
$\mathrm{API}$ & 31.4 \\
$\gamma_{g}$ & 0.67 \\
$\mathrm{P}_{b}(\mathrm{psi})$ & 487.2 \\
\hline
\end{tabular}

exact ANN algorithm used in this research is multi-layer perceptron (MLP) regressor [28]. To easily understand the MLP regressor, a sample artificial neural network architecture is shown in Fig. 2. There are three parts in the system: the input layer, the middle layer (also called the hidden layer) and the output layer. The input layer takes input from your datasets, and it is also the exposed part of the network. Often a neural network is drawn with an input layer with one neuron per input value or column in the dataset. It simply passes the input value through to the middle layer. Hidden layers are not
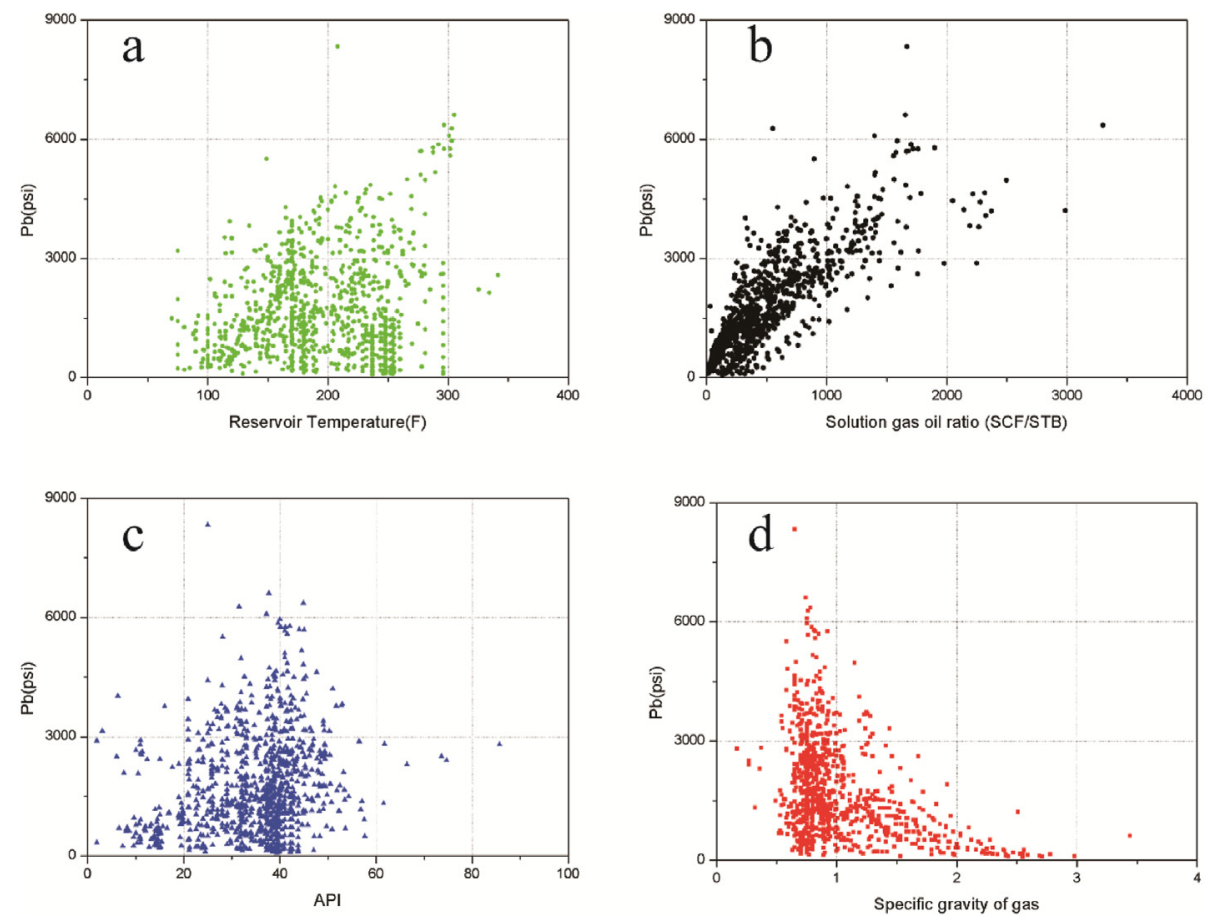

Fig. 3 - Saturation pressure distribution with each parameter ( a: Temperature; b: $\mathbf{R}_{\mathbf{s}}$; c: API; $d: \gamma_{g}$ ). 

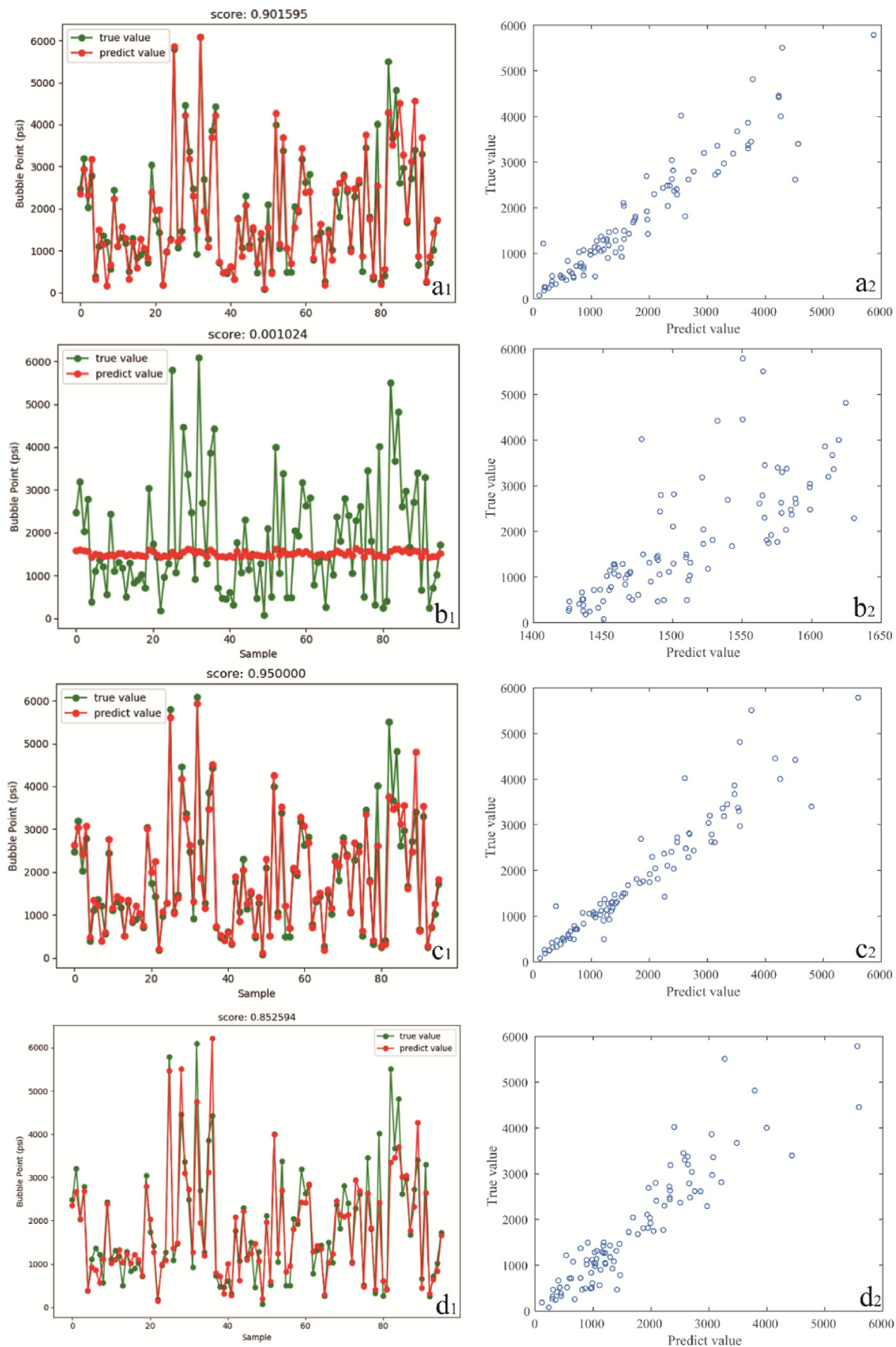

Fig. 4 - Correlation between true values and predicted value of saturation pressure by three methods. (a: DT; b: SVM; c: RF; d: NN).

directly exposed to the input. In a deep neural network, it has many hidden layers. Neurons or perceptron in the hidden layer that uses activation function to output a value for the next hidden layer or output layer. The output layer is responsible for outputting a value or vector of values for the exact problem. Usually the programmer could choose any numbers of hidden layers and the number of neurons in each layer. When there are many hidden layers and neurons in each layer, it will bring the possibility of over-fitting. In MLP regressor, $\mathrm{L} 2$ regularization is used to avoid overfitting in the ANN model. 

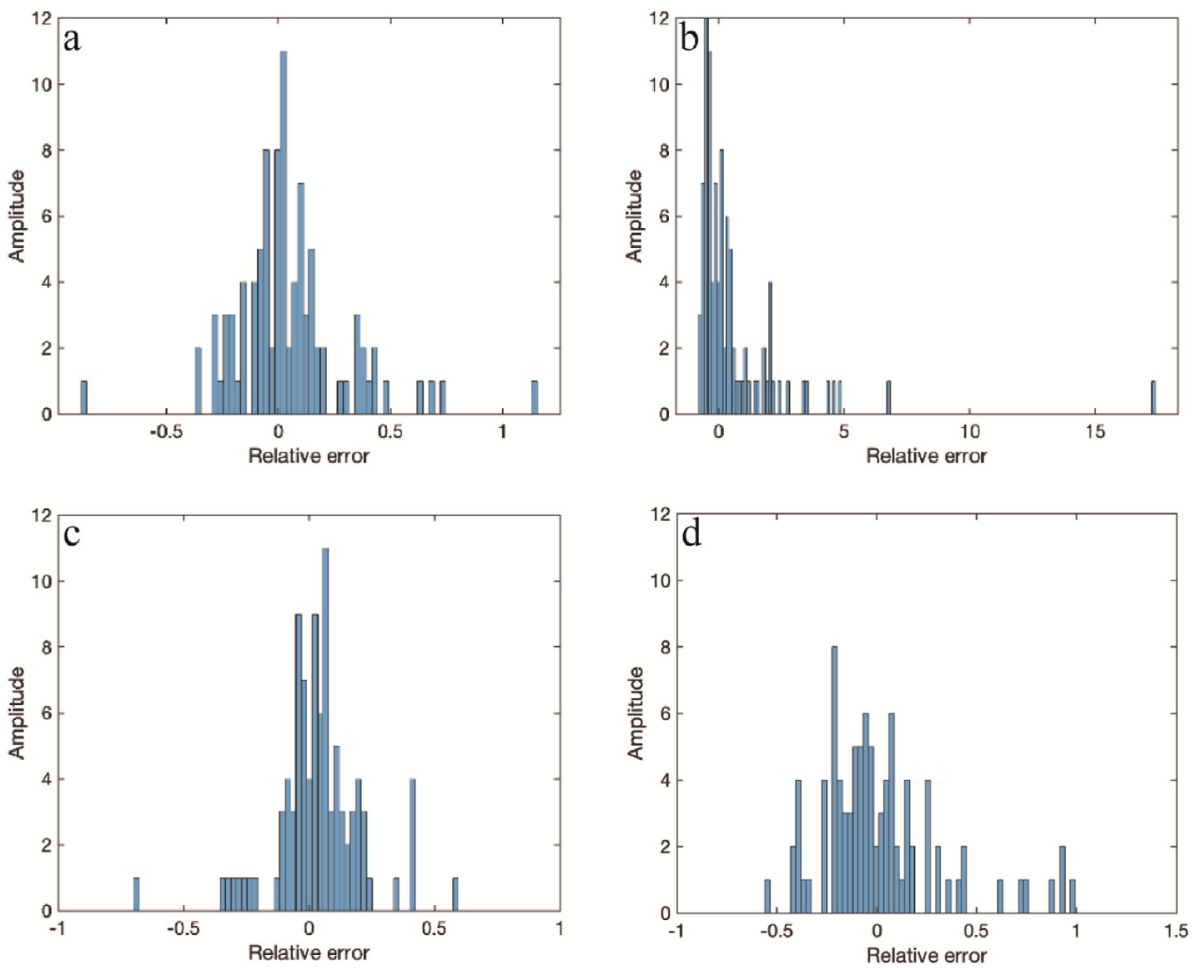

Fig. 5 - Prediction error distribution plots of data set. (a: DT; b: SVM; c: RF; d: NN).

\section{Results and discussions}

\section{Datasets}

Datasets used to implement the different algorithms were collected from several published sources and our company. These datasets consist of reservoir pressure, temperature, liquid hydrocarbon gravity, solution gas-oil ratio, and average gas gravity for calculating saturation pressure. A total of 940 datasets from about 7 different published papers were gathered and checked for quality. There are total 977 data sets with about 37 data sets from reservoirs in a west Africa basin unpublished in the literature. Data sources and the number of data sets collected from each source was shown in Table 1.

Data quality control is the first step before machine learning application. The data sets may contain typos, simple unit errors or some missing data which need to be fixed. The

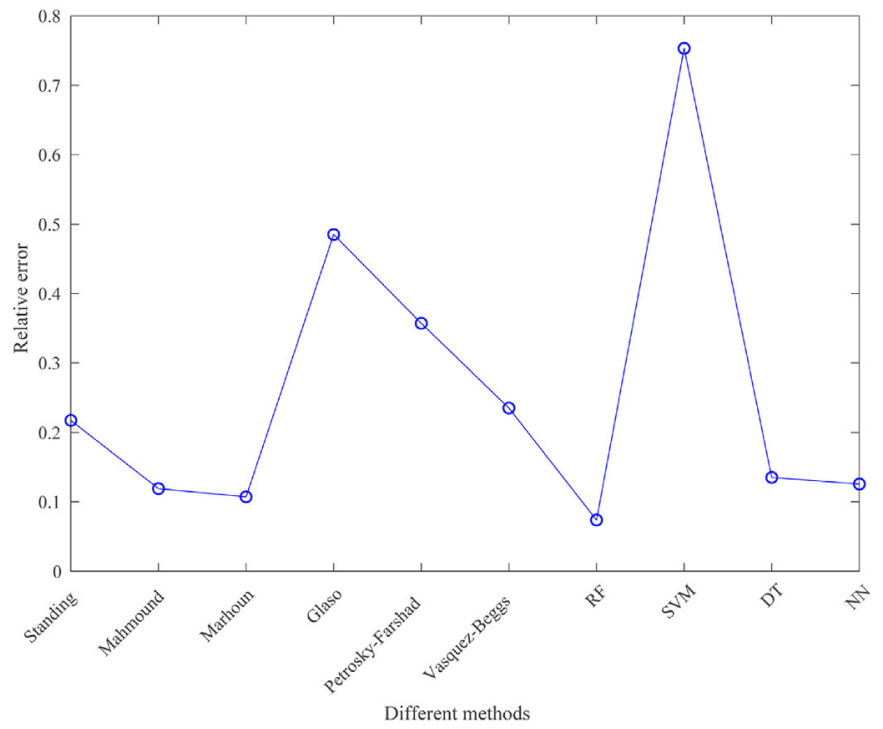

Fig. $6-P_{b}$ from $R F$ model compared with 6 common empirical correlations. 


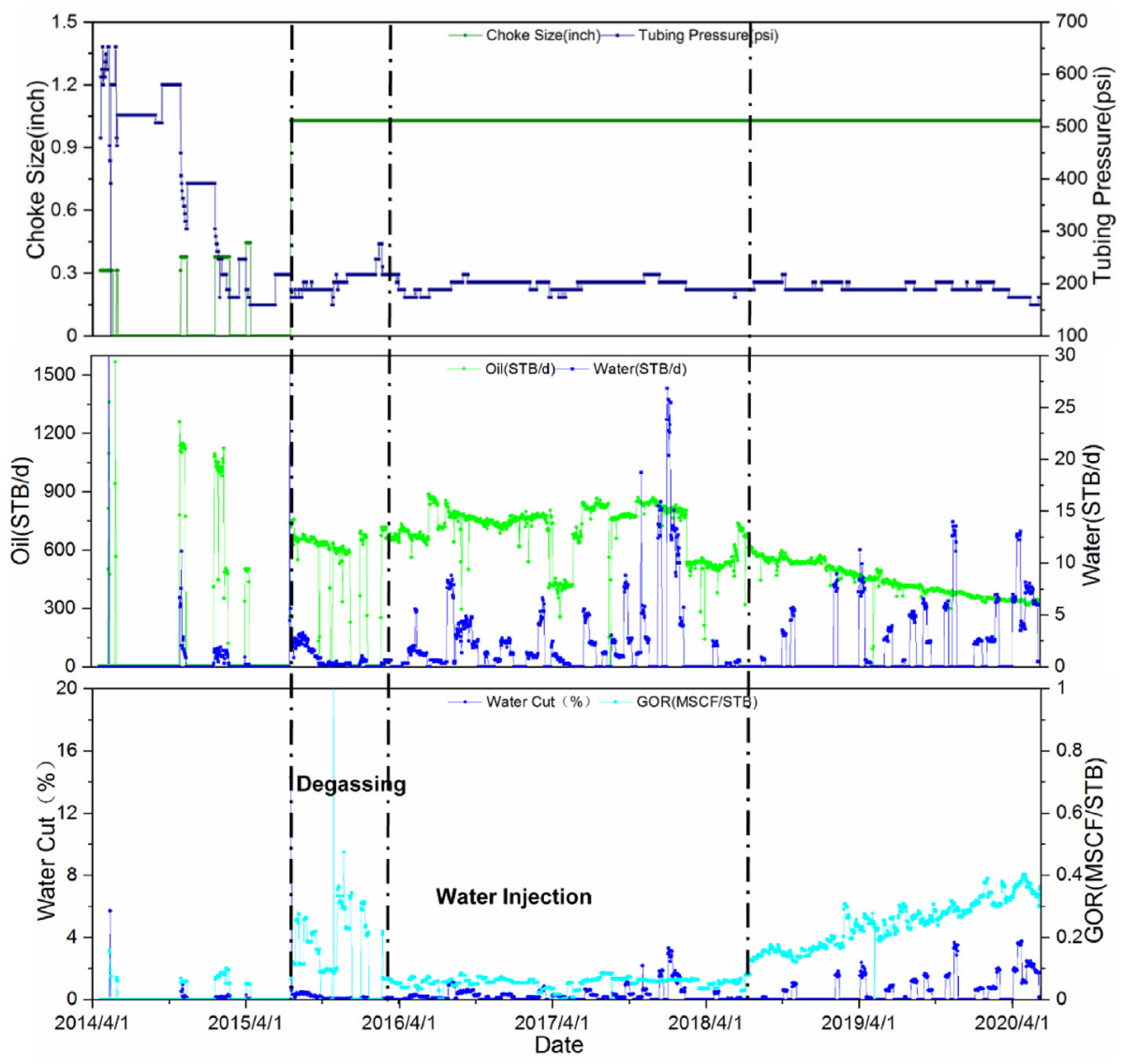

Fig. 7 - Well production curve.

inaccurate data set will give the wrong trained model to predict the correct value. As a result, the literature data after cleaning has 914 samples and the total number of training sets is 951. The statistical information is shown in Table 2 and Fig. 3. We split the data sets into two parts: training and test sets, the percentage about $90 \%-10 \%$ randomly. We use the training sets to train the random forest model and make some optimizations, and use the test sets to evaluate the model performance. Next is fitting random forest and training the model. We'll remove what is considered noise within feature space to reduce the inclusion of features, helping to improve

\section{Table 4 - Results from empirical correlations.}

\begin{tabular}{lc} 
Methods & $\mathrm{Pb}(\mathrm{psi})$ \\
\hline Standing & 593.1 \\
Mahmound & 429.2 \\
Marhoun & 435.0 \\
Glaso & 723.6 \\
Decision Tree & 421.4 \\
Vasquez-Beggs & 601.8 \\
Random Forest & 451.0 \\
SVM & 854.0 \\
PetroskyFarshad & 313.2 \\
Neural Network & 548.3 \\
\hline
\end{tabular}

the accuracy of the model. Cross-validation is used for estimating the predictive power of the RF model, and it performs better than the conventional training and test sets. In this case, the cross-validation score is 0.927 , showing that the RF model has the power and effectiveness to do the prediction.

We also use one dataset as the input to calculate the saturation pressure, and this set also has laboratory data. The input is shown in Table 3. After calculation, we get the predicted saturation pressure 451.3 psi from the RF model.

\section{Comparison of four prediction methods}

The prediction results calculated by RF, SVM, DT and ANN are shown in Fig. 4 left column. The correlations between the predicted value and measured value are demonstrated in Fig. 4 right column. It is clear that when RF algorithm is implemented, the prediction is the best. The QuantileQuantile plot illustrates that the predicted saturation pressure and measured saturation pressure had a great agreement with each other. The decision tree is the second-best method comparing to SVM. The SVM cannot accurately predict the saturation pressure value. The scatter plot shows that the tail points deviate from the line. Fig. 4 explains the prediction error distribution by histogram. The error of RF gives the Gaussian distribution with small standard deviation. 
However, the error of SVM has a big range of distribution which indicates the same results as results in Fig. 5. It can be seen that the RF model is a better method of predicting the saturation pressure, because it reduces the dependency between various variables significantly (see Fig. 6).

\section{Results from three methods}

Comparative studies were conducted to compare the performance of the RF model with SVM, DT and ANN methods and the 6 common empirical correlations (Standing, Mahmound, Al-Marhoun et al., Glaso, Petrosky-Farshad, and VasquezBeggs). SVM method has the highest value which is $854.2 \mathrm{psi}$, and Petrosky-Farshad method has the lowest value 313.2psi. The traditional empirical correlations method determines the coefficients by correlation in the formula, and only suit for specific area or reservoir, so it has a greater error. The details are shown in the Table 4 and Fig. 3.

Compared with lab test results and some empirical correlations, the performance of RF model for saturation pressure is very close to lab test and Marhoun correlation, with an average absolute error of about $10 \%$. There was only one well with saturation pressure test in this reservoir, and the data may also have an experimental and human error. Therefore, the model is acceptable, and the improvement in performance is expected with more datasets. When some wells have reservoir pressure, temperature, liquid hydrocarbon gravity, solution gas-oil ratio, and average gas gravity data, we do not spend extra money doing laboratory test to get saturation and can use the RF model to calculate it for the target reservoir.

Though the saturation pressure is too complicated, it's better to have more information from the reservoir. But when the experimental value of saturation pressure is not available. There is a need for the estimation of saturation pressure via the algorithms, such as RF model and other machine learning methods.

In this study we use many data from all over the world, each of them has different geological background and property, and we also split the data sets into two parts: training and test sets, the percentage about $90 \%-10 \%$ randomly, and the models have good score. So it can lower the effect of geological property on the saturation pressure prediction. It is reasonable to use machine learning to predict the saturation pressure.

\section{Application}

According to the methods above, the calculated saturation pressure is $507.5 \mathrm{psi}$. The measured flow pressure of the well on June 15, 2015, was 473.2 psi, which was lower than the predicted saturation pressure. There was degassed gas in the near-wellbore area and the gas-oil ratio was significantly higher (Fig. 5). For such thick reservoirs, which are easily degassed, they should be accurately monitored when they enter the dissolved gas stage and determine water injection time to maintain reservoir energy. This well group implemented water injection in April 2016, after that GOR had a descending trend and oil production rate became stable (Fig. 7).
If dissolved gas occurred in the reservoir, the reservoir may move to the secondary oil recovery stage, e.g., water injection or gas injection. The role of water injection mainly has the following functions: Firstly, it can supplement the reservoir energy, increase the reservoir pressure, and inhibit the degasification of crude oil in the formation; Secondly, the dissolved GOR increases gradually as the reservoir pressure increases, making natural gas from the formation re-dissolves into crude oil. After we get the predicted saturation pressure, we can use the following formula to calculate water injection rate to make sure the reservoir pressure higher than saturation pressure.

$\mathrm{W}_{\mathrm{i}}=\left(\mathrm{N}-\mathrm{N}_{p}\right) \mathrm{B}_{\mathrm{oi}} \mathrm{C}_{\mathrm{t}} \Delta p$

\section{Conclusion}

Based on the results of this study, the following conclusions can be obtained:

Compared with traditional methods, machine learning can lower the effect of geological property on the saturation pressure prediction. It is reasonable to use machine learning to predict the saturation pressure.

Compared with lab test results and some empirical correlations, the performance of the RF model for saturation pressure is very close to lab test, with an average absolute error.

This method is simple and accurate for crude oil saturation pressure, which lays a foundation for the next step reservoir research and development.

Water injection measures are proposed in this research. It can make up for the loss of formation energy, promote the recovery of reservoir pressure and inhibit the degassing of crude oil in the formation. The determination of the theoretical water injection rate provides a basis for the development of reasonable water injection in the oil field and evades the risk in the water injection process.

\section{Declaration of competing interest}

The authors declare that they have no known competing financial interests or personal relationships that could have appeared to influence the work reported in this paper.

\section{Acknowledgements}

This work was jointly supported by the Major Project of China National Petroleum Corporation (Grant No. 2016D-4402).

\section{Nomenclature}

API Stock-tank oil gravity, API

$B_{0} \quad$ Oil Formation Volume Factor, bbl/stb

$P_{b} \quad$ saturation pressure, psi

PVT Pressure Volume Temperature

$R_{S} \quad$ Solution gas-oil-ratio, scf/stb

$\mathrm{T}$ Temperature, ${ }^{\circ} \mathrm{C}$ 
$\gamma_{g} \quad$ Gas specific gravity (air $=1$ )

$\gamma_{0} \quad$ Oil specific gravity (Water $=1$ )

$\mathrm{W}_{\mathrm{i}} \quad$ Water injection rate, bbl ;

$\mathrm{N} \quad$ OOIP (original oil in place), bbl ;

$\mathrm{Np} \quad$ Cumulative oil, bbl ;

$B_{o i} \quad B_{g}$ at original oil pressure

$C_{\text {ot }} \quad$ Total compressibility, 1/psi ;

$\Delta p \quad$ Total pressure drop, psi

\section{R E F E R E N C E S}

[1] Lu K, Cai H, Wang L, Shi M, He X, Fan H. Establishment of an initial water production evaluation chart for the gas province in the East China Sea. Nat Gas Ind B 2019;6(6):580-7.

[2] Jiang T, Sun X. Development of Keshen ultra-deep and ultrahigh pressure gas reservoirs in the Kuqa foreland basin, Tarim Basin: understanding and technical countermeasures. Nat Gas Ind B 2019;6(1):16-24.

[3] Wang Ximeng, Liu Luofu, Wang Yang, Yue Sheng. Highpressure isothermal methane adsorption characteristic of Longmaxi Formation shale in the southern Sichuan Basin. Nat Gas Ind 2019;39(12):32-9.

[4] Zheng Lihui, Liu Hao, Zeng Hao, Wu Tong, Zhang Wenchang, Wang Chao. Evaluation of working fluid damage in fractured reservoirs using flow rate instead of permeability. Nat Gas Ind 2019;39(12):74-80.

[5] Du SH, Pang S, Shi YM. Quantitative characterization on the microscopic pore heterogeneity of tight oil reservoir by considering both the resolution and representativeness. J Petrol Sci Eng 2018a;169:388-92.

[6] Du SH, Pang S, Shi YM. A new and more precise experiment method for characterizing the mineralogical heterogeneity of unconventional hydrocarbon reservoirs. Fuel 2018b;232:666-71.

[7] Du SH, Xu F, Taskyn A, Zhou B, Kou G, Shi YM. Anisotropy characteristics of element composition in Upper Triassic "Chang 8" shale in Jiyuan district of Ordos Basin, China: microscopic evidence for the existence of predominant fracture zone. Fuel 2019a;253:685-90.

[8] Du SH, Zhao YP, Jin J, Kou G, Shi YM, Huang XF. Significance of the secondary pores in perthite for oil storage and flow in tight oil reservoir. Mar Petrol Geol 2019b;110:178-88.

[9] Du SH, Shi YM, Zheng XJ, Chai GS. Using "Umbrella Deconstruction \& Energy Dispersive Spectrometer (UD-EDS), technique to quantify the anisotropic elements distribution of" Chang 7" shale and its significance. Energy 2020;191:116443.

[10] Yi Xiangyi. The method of saturation pressure of predicting reservoir oil by calculation [J]. Journal of Xinjiang Petroleum Institute 1997;9(1):19-23.

[11] Standing MB. Volumetric and phase behavior of oil field hydrocarbon systems. Dallas, Texas: Society of Petroleum Engineers of AIME[J]; 1977.

[12] Petrosky GE, Farshad FF. Pressure-Volume-temperature correlations for gulf of Mexico crude oils. SPE Reservoir Eval Eng 1998;1(5):416-20.

[13] Glaso O. Generalized pressure-volume-temperature correlations[J]. J Petrol Technol 1980;32(5):785-95.

[14] Beggs HD, Vazquez ME. Correlation for fluid physical property prediction[J]. J Petrol Technol 1980;980:1.

[15] Bougher B, Herrmann F. AVA classification as an unsupervised machine-learning problem[M]//SEG Technical Program Expanded Abstracts 2016. Society of Exploration Geophysicists; 2016. p. 553-6.
[16] Noshi CI, Noynaert SF, Schubert JJ. Casing failure using machine learning algorithms: five case studies[C]//SPE thermal well integrity and design symposium. Society of Petroleum Engineers; 2018.

[17] Putcha VB, Ertekin T. A hybrid integrated compositional reservoir simulator coupling machine learning and hard computing protocols[C]//SPE Kingdom of Saudi Arabia Annual Technical Symposium and Exhibition. Society of Petroleum Engineers; 2018.

[18] Kellogg RP, Chessum W, Kwong R. Machine learning application for wellbore damage removal in the wilmington field[C]//SPE western regional meeting. Society of Petroleum Engineers; 2018.

[19] Zheng Junde, Sun Zhi, Sun Pinyue. Determination of oil saturation pressure correlations for Sazhong area Sapugao reservoir [J]. Petrol Explor Dev 1997;25(1):57-62.

[20] Nagi J, Kiong TS, Ahmed SK, et al. Prediction of PVT properties in crude oil systems using support vector machines[C]//Energy and Environment. In: 2009. ICEE 2009. 3rd international conference on. IEEE; 2009. p. 1-5.

[21] Moradi B, Malekzadeh E, Amani M, et al. Bubble point pressure empirical correlation[C]//Trinidad and Tobago Energy Resources Conference. Society of Petroleum Engineers; 2010.

[22] Alakbari FS, Elkatatny S, Baarimah SO. Prediction of bubble point pressure using artificial intelligence AI techniques [C]//SPE Middle East artificial lift conference and exhibition. Society of Petroleum Engineers; 2016.

[23] Svetnik V, Liaw A, Tong C, et al. Random forest: a classification and regression tool for compound classification and QSAR modeling[J]. J Chem Inf Comput Sci 2003;43(6):1947-58.

[24] Biau G, Scornet E. A random forest guided tour[J]. Test 2016;25(2):197-227.

[25] Belgiu M, Drăguţ L. Random forest in remote sensing: a review of applications and future directions[J]. ISPRS J Photogrammetry Remote Sens 2016;114:24-31.

[26] Burbidge R, Trotter M, Buxto B. Drug design by machine learning: support vector for pharmaceutical data analysis". Comput Chem 2001;26(1):5-14.

[27] Breiman L, Friedman J, Olshen R, Stone C. Classification and regression trees. Belmont, CA, USA: Wadsworth; 1984.

[28] Glorot X, Bengio Y. Understanding the difficulty of training deep feedforward neural networks. Proceedings of the Thirteenth International Conference on Artificial Intelligence and Statistics, PMLR 2010;9:249-56.

[29] Abdul-Majeed GHA, Salman NH. Statistical evaluation of PVT correlation-solution gas oil ratio. J Can. Pet. Tech. 1988;27(4):95-100.

[30] Bello OO. "Comparison of the performance of empirical models used for the prediction of the PVT properties of crude oils of the Niger delta," Petroleum science and Technology. Online Publication Date; 01 January 2008.

[31] De Ghetto, Giambattista and Villa, Marco: "Reliability analysis on PVT correlations," Paper SPE 28904 presented at the 1994 SPE European Petroleum conference, london, 25-27 October.

[32] Dokla, M. and Osman, M.: "Correlation of PVT properties for UAE crudes," SPE formation evaluation (march 1992) 4146; Trans, vol. 285, SPE Paper 21342.

[33] Mahmood MM, Al-Marhoun MA. Evaluation of empirically derived PVT properties for Pakistani crude oils. J. Pet. Sci. and Eng. 1996;16:275.

[34] Omar, M.I. and Todd, A.C.: "Development of new modified black oil correlation for Malaysian crudes," Paper SPE 25338 presented at the 1993 SPE asia pacific oil and gas conference, Singapore, 8-10 February. 
[35] Ostermann, R.D., Ehlig-Economides, C.A., and Owalabi, O.O.: "Correlations for the reservoir fluid properties of alaskan crudes," Paper SPE 11703 presented at the 1983 SPE California regional meeting, ventura, California, 23-25 March. 